\title{
Time for the epigenome
}

\author{
The complexity of genetic regulation is one of the great wonders of nature, but it represents a daunting \\ challenge to unravel. The International Human Epigenome Consortium is an appropriate response.
}

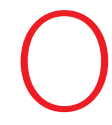
ncologist Ronald DePinho of Harvard University in Boston, Massachusetts, may be unaware of the impact of his innocent question at a meeting in 2004 on cancer epigenetics, a field then just starting to burgeon. But it was like the proverbial flap of a butterfly's wing in Brazil that caused a tornado in Texas. "Where do you want the field of epigenetics to be in five years?" he casually asked.

By 2004, large-scale genome projects were already indicating that genome sequences, within and across species, were too similar to be able to explain the diversity of life. It was instead clear that epigenetics - those changes to gene expression caused by chemical modification of DNA and its associated proteins - could explain much about how these similar genetic codes are expressed uniquely in different cells, in different environmental conditions and at different times.

That tiny perturbation - the curiosity of an interested outsider focused the thoughts of a few of the scientists there, overwhelmed by an explosion of new data, and spurred them to action. The next year they held a workshop under the auspices of the American Association for Cancer Research. The participants focused on the concept of the 'epigenome' - a genome-wide map of epigenetic modifications — and developed a blueprint for a Human Epigenome Project. This would reach much further than the epigenetics of cancer. Rather, it would amount to a global activity involving all relevant areas of biology. After all, given the universality of the fundamentals of gene regulation across all organisms and all cell types, biologists needed to be speaking the same language from the beginning.

This blueprint has been refined, and was launched last week as IHEC, the International Human Epigenome Consortium, the metaphorical tornado (see page 596). If DePinho were to ask his question now, the answer would trip instantly off the tongue: within a few years, epigeneticists will have hundreds of reliable reference epigenomes freely available to them at a mouse-click.

Although some may baulk at the launch of yet another giant '-omics' project, particularly in a complex area where there is still so much uncertainty, IHEC is in fact setting off at just the right time to instil order. It could be said, indeed, that the train has already left the station: the US National Institutes of Health is already setting up reference-epigenome centres, and the European Commission is planning to do so soon. This is not the time to reopen a clichéd debate on big science versus small science - the latter will carry on regardless, all the better for the standards IHEC will provide. It is instead the time for funding agencies, and, it is to be hoped, industry also, to generate money for an initiative that
"The project's most enthusiastic proponents refer to it as the hadron collider of biology." serves a vision that is both grand and thoroughly worthwhile.

IHEC's most enthusiastic proponents refer to it as the hadron collider of biology - an exuberant comparison that nonetheless bears consideration. CERN's Large Hadron Collider (LHC) near Geneva, Switzerland, is a global project searching for the most fundamental subatomic particles that must exist if the standard model of the physics underpinning our Universe is correct. If biologists were to refer to an equivalent standard model of the biological codes that underpin the diversity of life, the genetic code itself would be only a small part of it. Epigenetics, on the other hand, could contribute a very significant proportion of the rest.

In terms of sheer hardware, IHEC doesn't compare to the LHC. Nor will its costs be quite as huge. But its scientific vision is just as compelling. And, given that epigenetic coding will be orders of magnitude more complex than genetic coding, its requirement for data crunching may be similar. But IHEC will deliver information about health and disease as it progresses, particularly about intractable diseases such as cancer. For all of these reasons, and just as with the LHC, the Human Epigenome Project is a challenge whose time has come.

\section{Stand and deliver}

\section{Science has done well in the proposed US budget. Researchers need to justify the funding boost.}

\footnotetext{
T
} he surprises just keep coming. Even off the back of the worst economic crunch seen in many years, US President Barack Obama has evidently concluded that science should continue to be privileged. His US\$3.8-trillion budget request to Congress, unveiled on 1 February, contains generous funding for basic-science research, including a proposed $8 \%$ boost for the National Science Foundation and the largest percentage increase since 2003 for the National Institutes of Health (see page 594). Nearly every research agency emerges as a winner in Obama's budget proposals. Even NASA, heavily curtailed in its plans for human exploration of space, is slated for an increased spend on science.

The rises are remarkable given the political and fiscal stresses being experienced by the United States. Obama's administration has been trying to prove that it cares about responsible spending, and one focus of the proposed budget has been reining in the deficit, which is projected to reach up to $\$ 1.6$ trillion in the next year. The administration thus dutifully declared a freeze at present funding levels for most non-defence discretionary spending programmes - those that Congress has authority to change from year to year, which excludes major mandatory items such as social security and Medicare health-insurance programmes.

Because such discretionary spending accounts for about a seventh of the national budget, this freeze comes as something of a 